\title{
Addresses of reviewers
}

Kai-Henrik Barth, Universität Hamburg, Institut für Geschichte der Naturwissenschaften, Bundesstr. 55, D-2000 Hamburg 13

Dr. med. Thomas Böni, Lenzstr. 2, 8340 Hinwil

Dr. med. Karl Brändle, Badenerstr. 681, 8048 Zürich

Prof. Dr. phil. Johann Jakob Burckhardt, Bergheimstr. 4, 8032 Zürich

Dr. phil. Willem F. Daems, Stollenrain 15, Postfach 306, 4144 Arlesheim

Prof. Dr. Charles P.Enz, Ph. D., Département de physique théorique, Université de Genève, 24, quai Ernest-Ansermet, 1211 Genève 4

Dr. phil. Hans-Rudolf Fehlmann-Aebi, Quartierweg 18, 5115 Möriken

Prof. Dr.med.Stephanos Geroulanos, Departement Chirurgie, Universitätsspital, 8091 Zürich

Dr.phil. Beat Glaus, ETH-Bibliothek, Rämistr. 101, 8092 Zürich

Prof.Dr. med. Carl Haffter, Petersgasse 50, 4051 Basel

Dr. Klaus Hentschel, Cankebeerstr. 97, D-2988 Westdorf/Nesse 1

Dr. med. Hansruedi Isler, Neurologische Klinik und Poliklinik, Frauenklinikstr. 26, 8091 Zürich

Dr. med. Hans Urs Keller-Schnider, Mühleackerstr. 539, 4707 Deitingen

Dr. Claus Kiefer, Institut für Theoretische Physik, Universität Zürich, Schönberggasse 9, 8001 Zürich

Prof. Dr.med. Huldrych M. Koelbing, Gotthardstr. 65, 8002 Zürich

PD Dr. François Ledermann, Schloss-Apotheke, Könizstr. 3, 3000 Bern 21

Prof. Dr. med. Jean Lindenmann, Institut für Immunologie und Virologie, Universität Zürich, Gloriastr. 30, Postfach, 8028 Zürich

Prof. Dr. phil. Innocenzo Mazzini, Via A. Gianandrea, 8, I-60035 Jesi (An.)

Prof. Dr. med.Christian Müller, Herrengasse 23, 3011 Bern

PD Dr. phil. Erwin Neuenschwander, Mathematisches Institut, Universität Zürich, Rämistr. 74, 8001 Zürich

PD Dr. phil. Ueli Niederer $\dagger$, Remigerstr. 243, 5223 Riniken

Dr. med. Markus Oettli, Hauptstr.31, 8586 Erlen

Prof. Dr. med. Hans Remky, Arabellastr. 5, D-8000 München 81

Dr. med. René Renggli, Largitzenstr. 77, 4056 Basel

Dr. med. Kurt Ritzmann, Schwalbenbodenstr. 5a, 8832 Wollerau

Lic.phil.Sabina Roth, Historisches Seminar, Universität Zürich, Minervastr. 51, 8032 Zürich

Prof. Dr. med.Gerhard Rudolph, Jamm-Str.4, D-7630 Lahr

Dr. phil. Hans Konrad Schmutz, Naturwissenschaftliche Sammlungen, Museumstr. 52, 8400 Winterthur

PD Dr. Dr. Gottfried Schramm, Am Brunnenbächli 12, 8125 Zollikerberg

Dr. med. dent. Guido Sigron, Aathalstr. 19, 8610 Uster

Dr. phil. Verena Stadler, Hegibachstr. 149, 8032 Zürich

Dr. med.Antoinette Stettler, Kirchenfeldstr. 45, 3005 Bern

Prof. Dr. phil. Bartel Leendert Van der Waerden, Wiesliacher 5, 8053 Zürich

Dr. Burghard Weiss, TU Berlin, Institut für Philosophie, Wissenschaftstheorie, Wissenschaftsund Technikgeschichte, Ernst-Reuter-Platz 7, D-1000 Berlin 10

Prof.Dr.med.Gerhard Zbinden, Institut für Toxikologie der ETH und Universität Zürich, Schorenstr. 16, 8603 Schwerzenbach 